\title{
THE INTENSITY OF NATURAL SELECTION IN MAN.
}

\author{
SECOND PAPER.
}

BY E. C. SNOW, M.A., D.Se.

THE present paper is a supplement to the memoir of the same title issued last year*. It is not proposed to give here any account of the work which has been done in the attempt to elicit information on the more difficult subject of the nature of selection in man, but only to publish the correlations and regressions obtained by using an alternative measure of environment, and by varying the periods in which the effects of a selective death-rate can be detected.

The adoption of another method of correcting for environment implies not the slightest shaking of my confidence in the adequacy and validity of that employed in the first memoir, but the further work was entered upon because the importanee of the subject renders the comparison of the results reached by the use of the various possible methods particularly desirable. The only criticism I have seen of the mode of measuring environment used in the earlier work is that by the Editors of the Journal of the Royal Statistical Society. Had there been a tithe of evidence supporting the view adumbrated in that criticism the memoir would have been practically valueless. Fortunately, however, no arguments whatever have been put forward in favour of the view held by the statistical critics, and very cogent facts against that view have already been given $\dagger$. It is quite beside the point to show that the corrected standard deviation of the total mortality in the two periods considered is only $5 \%$ or $6 \%$. That standard deviation is in a number of cases appreciably of the same magnitude as the corresponding measure of dispersion in the earlier of the periods used, and, ruoreover, is many times its probable error. It matters not whether that standard deviation be $6 \%$ or ${ }^{\circ} 06^{\circ} \%$ or $60 \%$ of the mean value.

* Drapers' Company Research Memoirs, "Studies in National Deterioration," No. VII. Dulau and Co. 1911.

† Biometrika, Vol. vi1., p. 456, 1912. 
The notions used to indicate environmental conditions for this new work are very simple. Shortly, they consist in making the mortality of a cohort of one sex the measure of the environment for the corresponding cohort of the opposite sex on which it is desired to ascertain the possible effects of selection. Thus if we wish to investigate the selective effect on the male mortality of the third, fourth and fifth years of life of variations in that mortality in the first two years, in addition to fixing the size of the male cohort we fix the size of the corresponding female cohort and also the total female mortality in the first five years of life. We can thus suppose that we are dealing with districts in which the female mortalities up to five years of age for the cohorts born in a particular year are the same. For these districts we find varying male mortalities in each of the periods considered (see Table below), and the mean values of these male mortalities in both periods throughout the whole series of districts can be found. Do the mortalities in the second period of those districts whose mortalities in the first period deviated in the positive direction deviate, on the average, in the positive or negative direction? Districts with the same female environment will possess varying proportions of male weaklings. If these weaklings are killed off in the earlier period, the population which survives to the later one is stronger and likely, therefore, to have a smaller mortality, and this would be indicated by a negative correlation between the mortalities in the two periods (with the proviso dealt with in $\S$ XxxIV of the memoir). To the criticism that the total male mortality is highly correlated with the total female mortality, and that by making the latter constant we are practically fixing the former, we can reply by pointing to the considerable standard deviation of the total male mortality when correction is made for constant female mortality (see Table below). Evidence of a more gencral character, too, can be gathered by turning over the leaves of any of the RegistrarGeneral's valuable Decennial Supplements to his Annual Reports. Pick out a few of the registration districts in which the mortality of one sex for any of the age-groups given is practically the same and compare the mortalities of the other sex among those districts for the same age-group. Quite appreciable variation in the numbers will be found to exist. Not many of such districts can actually be found, but the method of partial correlation essentially consists of a contrivance by which we can for statistical purposes reduce all districts to a constant type.

No method of measuring environment can be theoretically perfect. Districts under the same environmental conditions would not have the same mortality, but the latter would be distributed in some way due to random causes. Of two districts under the same general environment one may in a particular year suffer to a greater extent from epidemics of measles, scarlet fever or summer diarrhœa, and part of the problem of selection consists in ascertaining if these epidemics strike more at the weaker children than at a random sample of all children, and this is ascertained by inquiring if the surviving population is more immune in a subsequent period. The two methods which are employed in this paper to measure environment are quite distinct and bear no physical relation to each other. In the one 
method the mortality of the cohort corresponding to the cohort whose history is being traced but of the opposite sex, is fixed. In the other, the number of deaths of the same sex and between the same age limits in the period under notice, apart from the deaths occurring within the cohort, is rendered constant. Neither of these, of course, allows for the fact that under a perfectly uniform environment the mortality which is taken to indicate that environment should be distributed in some way due to random causes. But the general similarity which will be shown to exist between the results reached by using the two distinct methods is some justification for the claim that each is a satisfactory approximation to the theoretically best method.

The investigation which is now being described was directed throughout to ascertaining the extent of selection in the mortality of the first two years of life. In the earlier work of which an account is given in the memoir other periods were taken, but later some evidence was adduced to indicate that the first two years of life was a natural interval to adopt, as embracing roughly the whole of the mortality of infancy and overlapping but very little that of childhood. For the second period, on the mortality of which the selective character of that of the earlier period is indicated, the next three years of life are taken both for the English and Prussian data; in the case of the latter, also, the next eight years are employed as a second period. We thus reach results obtained from English and Prussian data by working at the same periods in each case, and the comparison of these results is of interest. The notation employed throughout is:

$x_{0}=$ Births of the male or fernale cohort considered, in, say, year $t$.

$x_{1}=$ Deaths in the cohort in the two years, $t$ and $t+1$.

$x_{2}=$ Deaths in the cohort in the next three years, or next eight years.

$x_{3}=$ Remaining deaths of same sex as cohort in the five years or ten years (see Memoir, $\S$ vili).

$x_{4}=$ Deaths in the corresponding cohort of opposite sex in the five years or ten years considered.

$x_{5}=$ Births of the corresponding cohort of opposite sex.

Previous experience suggested that the correction for a constant value of $x_{5}$ in addition to constant values of $x_{0}$ and $x_{4}$ would have little effect on the correlation between $x_{1}$ and $x_{2}$. The first case worked out supported this view; in that (the Prussian male cohort of 1881, dealing with the first ten years of life) the correlation was only altered in the sixth figure by the extra correction, viz. from -944206 to -944209 . Thus the considerable labour involved in making this further correction is not justified by the extra value it gives to the results, and in all the other cases it was omitted.

The partial standard deviations, correlations and regressions with their probable errors for the various sets of data are given below, the other standard deviations and correlations on which they are based being shown at the end. In the table 
Males.

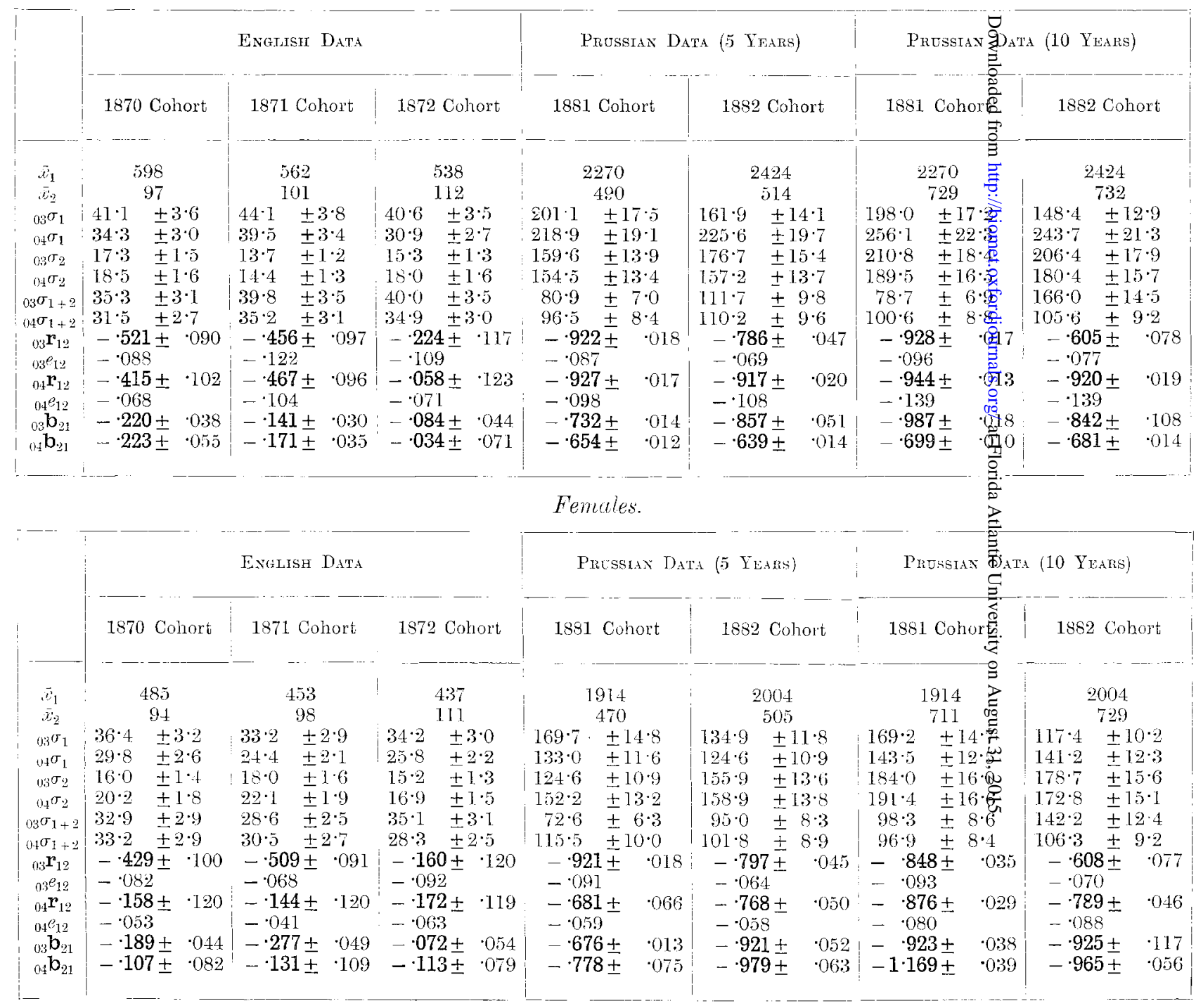


on p. $61{ }_{03} \sigma_{1+2}$ denotes the partial standard deviation of the total mortality in the sum of the periods considered, ${ }_{03} e_{12}$ denotes the expected (partial) correlation if there were no selection (see $\S \times x x I v$ of the memoir), and ${ }_{03} b_{0_{11}}$ denotes the (partial) regression of the mortality of the second period on that of the first.

Dealing first with the results from the English data, we notice that the regressions by the two methods for males are fairly similar, but for females they are, on the whole, smaller by the new mothod. Having regard to the probable errors we can draw no inferences concerning the differences. The correlations fur females, however, are in two of the three cases consilerably smaller by the new measure of environment, and this difference appears to be significant. The partial standard deviations by the two methods occasionally show fairly large differences, but in no single case is the disagreement significant. For all the six cases, however, ${ }_{01} \sigma_{1}$ is less than ${ }_{03} \sigma_{1}$ but ${ }_{04} \sigma_{2}$ is greater than ${ }_{03} \sigma_{2}$. The mean of the male regressions by the first method of measuring environment is -142 and by the other -143 , the corresponding figures for females being -179 and $-\cdot 117$. The mean regressions of the mortality of the 4th and 5th years of life on that of the first three years are -.085 and -.172 respectively*. Thus, so far as males are concerned, the intensity of selection appears greater when measured by the regression of the mortality of the 3rd, 4th and 5th years on that of the first two years of life than when measured by the regression of the mortality of the 4th and 5th over the first three years, but the same conclusion does not so definitely hold for females. It may be, as was suggested in the memoir, that the age division between infant and child mortality is not the same for females as for inales, and the inference is put forward tentatively that the ailments of infancy (as distinct from those of childhood) attack females to a rather greater age than they do males. It will be noticed, too, that the regressions and correlations for the 1872 cohort are smaller than for the other cohorts, and that this is accompanied by the fact that the mortality of that cohort was smaller in the first period. On the whole, so far as the data for the English rural districts are concerned, the adoption of a new measure of environment leads to no alteration of view as regards the existence of selection, nor, roughly, of its numerical intensity.

When we turn to the results from the Prussian data in which the same periods (the first two years and the next three) are used as for the English data, the most marked feature to be noticed is the considerably larger correlations and regressions which are obtained. The mean value of the regressions for males

* I take here the opportunity of correcting a mistake which occurred in connection with the work of the first memoir, through an error in transcribing from the schedules containing the raw data. In $\S$ xvir, in the portion of the Table for the 1872 cohort referring to females, the following alterations should be made:

$$
\bar{x}_{1}=487, \quad \sigma_{1}=119 \cdot 7,
$$

$r_{01}=812328, \quad r_{12}=\cdot 718137, \quad r_{13}=.944379, \quad{ }_{3} r_{01}=+\cdot 464859, \quad{ }_{3} r_{12}=-\cdot 168670, \quad{ }_{103} r_{12}=-\cdot 184068$

These entail the following corrections in the Table on p. 33 for the same cohort: $03 \sigma_{1}=34 \cdot 844$ and partial regression $=-0591$. 'Jhese alterations reduce the correlation and regression but make the results more consistent, and necessitate little modification of the conclusions drawn from them. 
is -.794 by the first method and -.647 by the second, while for females the figures are - .798 and -.878 , these comparing with corresponding values for the English districts ranging from -.12 to $-\cdot 18$. Thus the criterion which we take as the measure of the intensity of selection was for the Prussian cohorts of 1881 and 1882 about five or six times as large as that for the English cohorts of 1870 , 1871 and 1872. We can assert with some confidence a considerably greater selective effect of the mortality of the first two years of life on that of the next three in the case of Prussian rural districts than of the English rural districts in the epochs considered, and this fact is concomitant with a far greater stringency of infantile conditions in the former than in the latter. This is seen from the following figures.

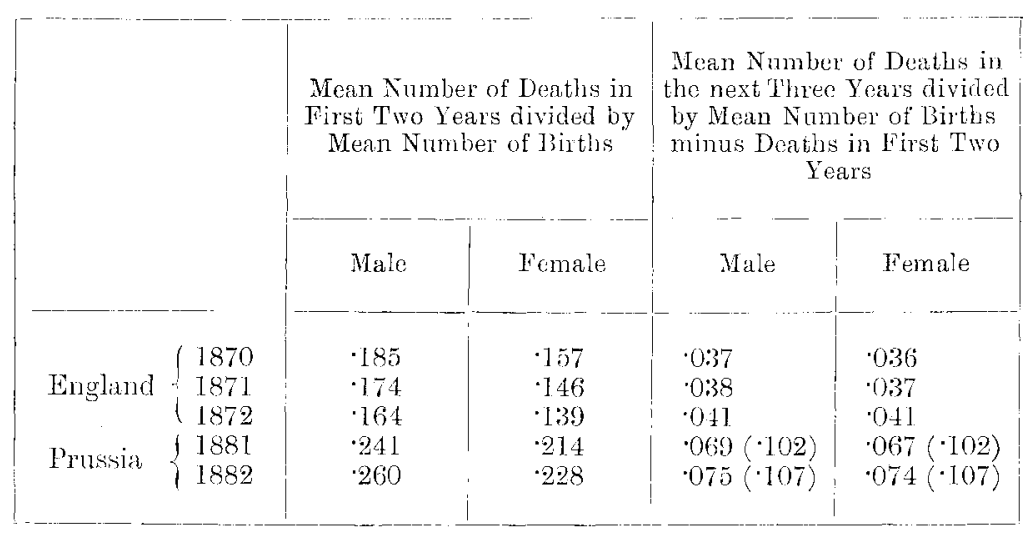

The figures in brackets give the corresponding numbers for Prussian districts for the mortality in the eight ycars following the first two.

The data for English rural districts do not allow us satisfactorily to follow the cohorts beyond their first five years of life, so that we cannot assert that the intensity of selection is generally less in Engiand than in Prussia for the populations considered, but we can point out definitely that the effect of selection in the first five years of life was much grcater in the latter country than in the former. Whether or not the English cohorts make up the leeway at later ages can only be a matter of speculation. We can at present merely state that whereas a district in England which had an excess of 100 male survivors above the mean for all districts at the end of the first two years of life had, on the average, about 14 of these survivors killed off in the next three years, a similar district in Prussia lost more than 70 of the 100 in the same period.

When, for the Prussian data, we come to deal with the results of including the eight ycars following the first two, we find that the regressions for the 1881 cohort have increased appreciably, but those for the 1882 cohort have not done so. There is nothing incongruous in this, as in one cohort selection might well be felt more in the 3rd, 4th and 5th years than in the other, and in this latter the effect would 
then probably come in later years. The tendency of the partial standard deviations for the Prussian data is opposite to that for the English, viz. for males ${ }_{04} \sigma_{1}$ is greater than ${ }_{03} \sigma_{1}$ and ${ }_{04} \sigma_{2}$ is less than ${ }_{03} \sigma_{2}$. For females, however, these are reversed, except for the 1882 cohort in the ten-year period. Another feature of the Prussian results is that the regressions for males are smaller by the new method of measuring environment, but for females the reverse is the case. This is also true for four out of the six examples from English data, and arises chiefly from the differences in the male and female variability in mortality in the second period compared with the first. In the first two years of life the (partial) standard deviation for males is always greater than for females, but for the second period the female (partial) standard deviation is in some cases the larger, the mean mortality in this second period being about the same for the two sexes.

In the memoir ( $\S$ XXIV) a short discussion is given to the question of what amount of correlation between the mortalities should be expected if selection were entirely absent. This has been referred to as ${ }_{03} e_{12}$ and ${ }_{04} e_{12}$ in the present paper. These values are only intended as approximations, and it would undoubtedly be an advantage if by direct correlations we could obviate the use of such corrections. 'These direct values could be obtained by correlating the mortality rate of the first two years of life (based on the number of births) of the cohort with the mortality rate in the 3rd, 4th and 5th (or 3rd to 10th) years of life (based on the number of survivors to the age of two), correction being made in some manner for a constant environment rate. 'This would entail a correlation between such variables as $\frac{x}{y}$ and $\frac{z}{x-y}$ and in my opinion might involve an element of 'spurious' correlation, and for this reason alone rates were not used in the memoir. So far as I can understand, however, the erities of the memoir do not hold this opinion, so that to them the corrected correlation between two variates of the above type is probably as satisfactory as a partial corrclation of the third order. The employment of such correlations saves considerable labour and requires no discussion of the question of 'expected' correlation if selection were inoperative. Accordingly, for a few cases, the following new variables have been taken:

$z_{0}=$ Male or Female Deaths in the first two years of life divided by Male or Female Births,

$z_{1}=$ Male or Female Deaths in next three (or eight) years of life divided by number of survivors to the age of two,

$z_{2}=$ Total Female or Male (i.e. of opposite sex to $z_{0}$ and $z_{1}$ ) 1)eaths in the whole five (or ten) years divided by Female or Male Births,

and the values of ${ }_{2} r_{01}$ worked out. The statistical constants on which they are based are given below: 


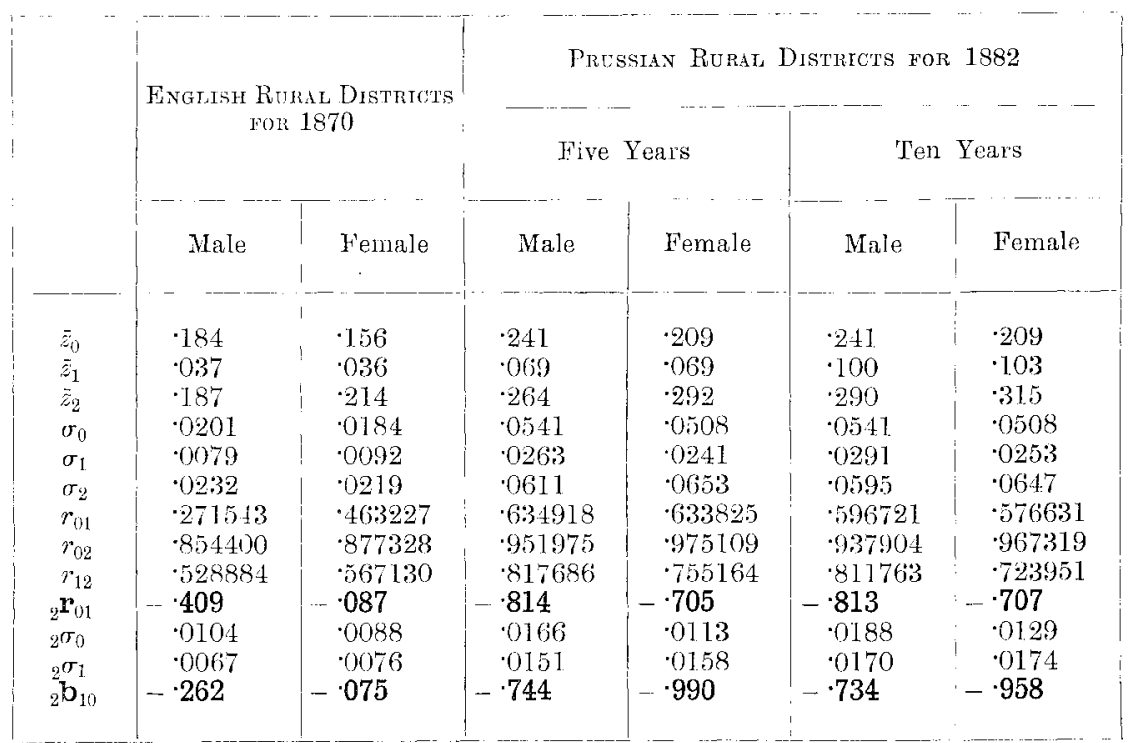

If the values of ${ }_{2} r_{01}$ here are compared with the values of ${ }_{04} r_{12}$ in the earlier tables for the corresponding cases we find very little difference. Thus:

Correlations obtained without using Rates,

$$
\begin{array}{llllll}
-.415 & -.158 & -.917 & -.768 & --.920 & -.789
\end{array}
$$

Corresponding Correlations by use of Rates,

$$
\begin{array}{llllll}
-409 & -.087 & -.814 & -.705 & -.813 & -.707
\end{array}
$$

If we reduce the figures in the first of these by the values of ${ }_{0 i} e_{12}$ we have the sequence

$$
\begin{array}{llllll}
-347 & -.105 & -.809 & -.710 & -781 & -701
\end{array}
$$

The agreement between the last two lines of figures is surprising and remarkable. In all cases we get approximately the same numbers as before, a quite unexpected result.

Lest these results should hastily be pointed to as evidence against the possibility of spurious correlation arising when such variables as $\frac{x}{y}$ and $\frac{z}{x-y}$ are correlated, it should be pointed out that the deviations from the mean values of the variables are in some cases considerable, and that the third and fourth powers of those deviations cannot be neglected in comparison with the corresponding powers of the means. Thus the formula which is usually exhibited to show the possibility of spurious correlation does not apply to this case. Whatever the magnitude of the 'spurious' element, if any, involved in the correlations just, found, they can only be construed as supporting those previously found and as evidence of the existence of selective mortality in the populations dealt with.

Professor Pearson, also, has pointed out to me another possible mode of attacking the problem. This is to render constant $x_{0}-x_{1}$ instead of $x_{0}$, and in 
addition to $x_{3}$ or $x_{4}$, as before. This would fix, not the number of births, but the number of survivors at the end of the first two years. Then the population liable to mortality in the second period would be the same for all districts, but the districts with the larger number of births would be exposed to the possibility of greater mortality in the first period, and therefore to the possibility of greater mortality of the kind which is taken to measure environment (the mortality in the first two years being much greater than in the next three or next eight). Thus an 'expected' (negative) corrclation if selection were absent would not arise in the same manner as before, but would probably be entailed in the partial correlation. A priori, however, it does not appear that this method would produce a correlation, if selection were inoperative, of the same magnitude as that indicated in $\$$ XXIV of the memoir, since the population rendered constant stands in an intermediate position to those at the beginuing and end of the periods considered. The correlations under these new conditions have been worked out only for the case of the 1884 Prussian cohort, both male and female. The results differ but very little from those reached before, and must, $\mathbb{I}$ think, be taken as supporting the substantial accuracy of the interpretation put upon the earlicr ones. If $x_{5}$ denote $x_{0}-x_{1}$, the following are the additional correlations:

\begin{tabular}{|c|c|c|}
\hline & Males & Fiemales \\
\hline$\sigma_{5}$ & $3117 \cdot 6$ & $3175 \cdot 1$ \\
\hline$r_{51}$ & .924739 & .928746 \\
\hline$r_{i 2}$ & $.8471: 38$ & .851981 \\
\hline$r_{53}$ & .924711 & $-9 ! 2791$ \\
\hline$r_{54}$ & $\cdot 927020$ & .934079 \\
\hline$\therefore \mathbb{I}^{2}$ & -.918 & -.747 \\
\hline $5+11^{\circ} 12$ & -.780 & -.806 \\
\hline
\end{tabular}

The values previously found for ${ }_{03} r_{12}$ and ${ }_{04} r_{12}$ were -.917 and -786 for males, and -.768 and -.797 for females. It appoars therefore to be of little account whother we make $x_{0}$ or $x_{0}-x_{1}$ measure the size of the populations; we should probably, too, got similar results if we put $x_{0}-x_{1}-x_{2}$, i.e. the size of the cohorts at the end of our survey, constant.

On the whole, the work of which this paper gives a short account has justified itself by the confirmation and emphasis it gives to the results previously obtained. The general impression received by a study of the results reached by the employment of the new method of measuring environment alone is much the same as that derived from a survey of those by the earlier one, though individual differences of appreciable magnitude occur. Apart from the cmphasis it gives to the results of the memoir, the present work has discovered, $I$ think, a significant difference in the operation of selection on the mortality of the first five years of life in Prussian and in English rural districts, and suggests (but, at present, no more than suggests) that there is some differentiation in its effect upon the two sexes. But the existence of a sclective death-rate in the general populations dealt with admits of no doubt. 
List of Statistical Constants on which tme Partial Correlations AND Regressions ARE BASED.

\section{English Rural Districts.}

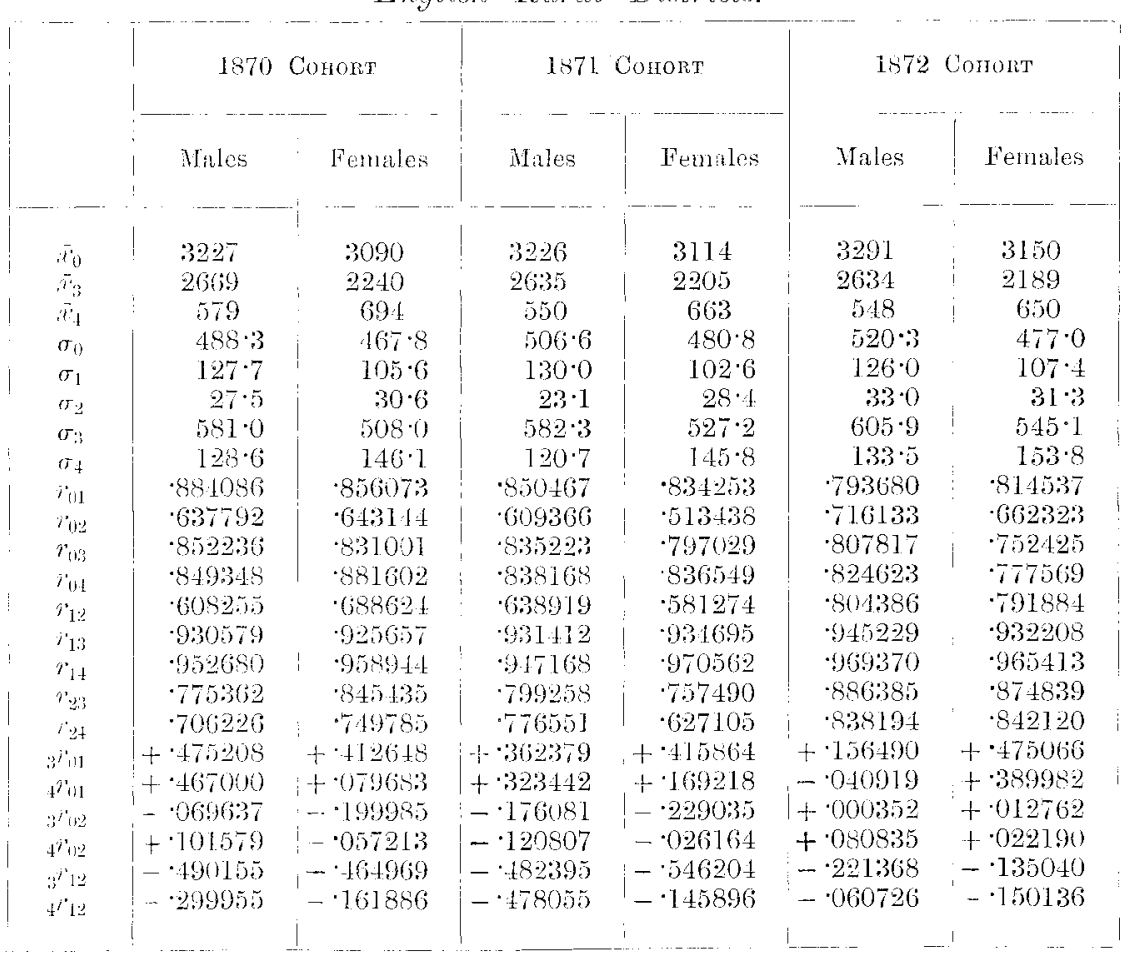

Prussian Rural Distriots (Five year period).

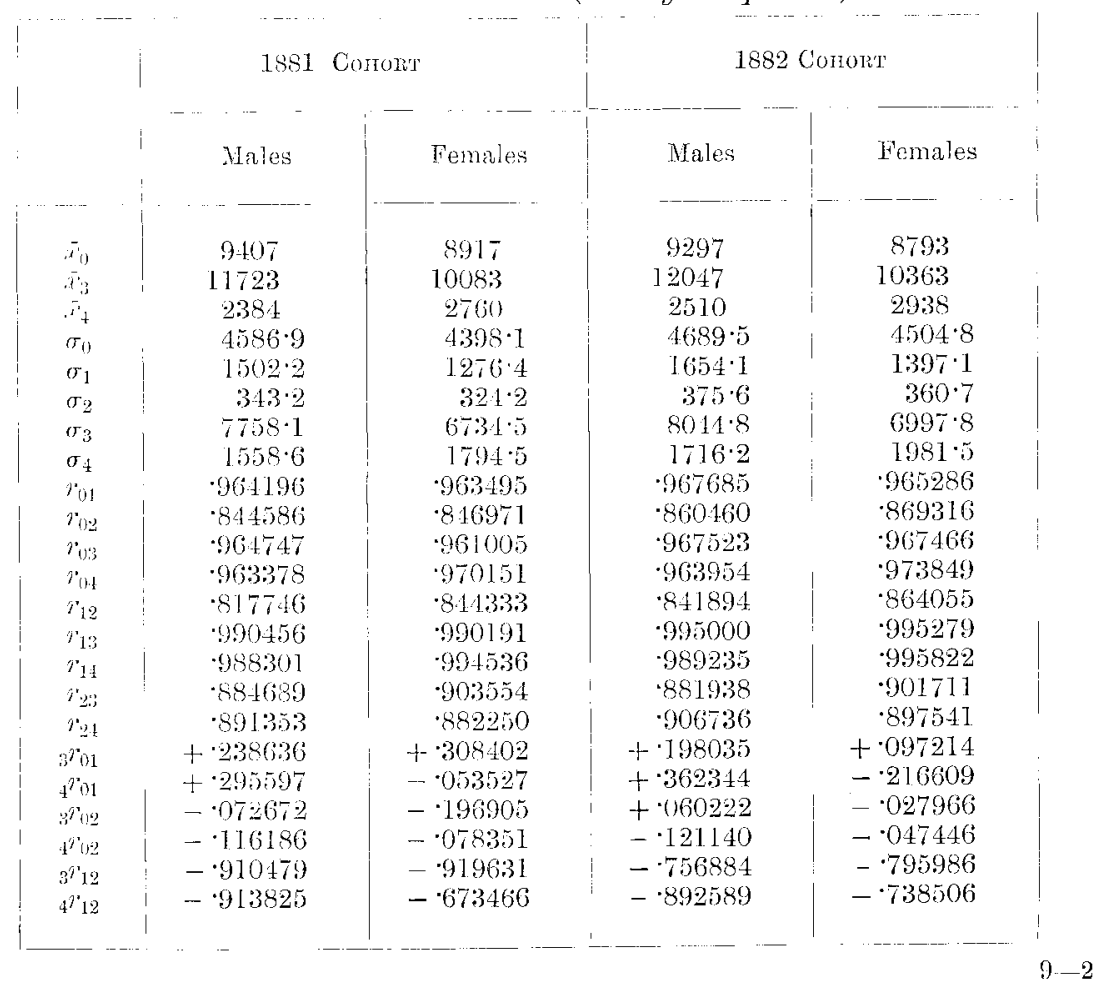


Prussian Rural Districts ('T'en-year period).

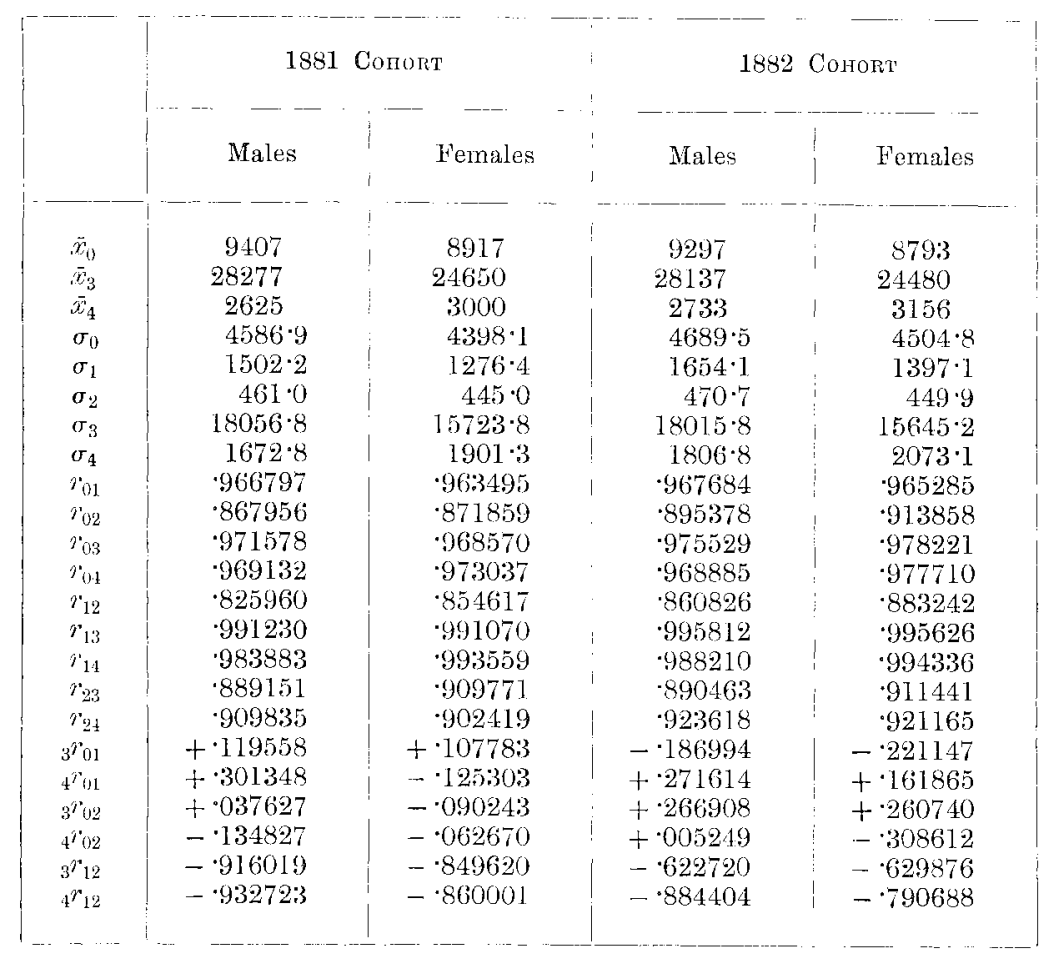

Also for the 1881 male cohort:

$$
\begin{aligned}
& r_{05}=.999225, \quad r_{15}=.965417, \quad r_{25}=.866632, \\
& r_{\mathrm{s} \overline{5}}=967678, \quad{ }_{3} r_{155}=+.987800, \quad{ }_{3} r_{15}=+\cdot 295690, \\
& { }_{3} r_{25}=-131819, \quad{ }_{03} r_{15}=-013340, \quad{ }_{03} r_{25}=+{ }^{\circ} 0088333 \text {, } \\
& { }_{035} r_{12}=-944209 .
\end{aligned}
$$

